\title{
Assessment of apoptosis and MMP-1, MMP-3 and TIMP-2 expression in tibial hyaline cartilage after viable medial meniscus transplantation in the rabbit
}

\author{
Tomasz J. Zwierzchowski ${ }^{1}$, Olga Stasikowska-Kanicka², Marian Danilewicz², Jarosław Fabiś ${ }^{1}$
}

${ }^{1}$ Arthroscopy and Sports Traumatology Clinic, Orthopedic Department,
Medical University of Lodz, Poland

2Department of Nephropathology, Medical University of Lodz, Poland

Submitted: 19 July 2011

Accepted: 2 January 2012

Arch Med Sci 2012; 8, 6: 1108-1114

DOI: 10.5114 /aoms.2012.30947

Copyright (c) 2012 Termedia \& Banach

\section{Abstract}

Introduction: The porpuse of this animal study was to assess chondrocyte apoptosis and MMP-1, MMP-3 and TIMP-2 expression in rabbit tibial cartilage 6 months after viable medial meniscal autografts and allografts.

Material and methods: Twenty white male New Zealand rabbits were chosen for the study. The medial meniscus was excised from 14 animals and stored under tissue culture conditions for 2 weeks, following which t of them were implantated as autografts and 7 as allografts. The control group consisted of 6 animals which underwent arthtrotomy. When the animals were eutanized, the tibial cartilage was used for immunohisochemical examination. Apoptosis (TUNEL method) and MMP-1, MMP-3 and TIMP-2 expression were estimated semiquantatively.

Results: An increased level of chodrocyte apoptosis in the tibail cartilage was observed after both kinds of transplants $(p<0.05)$, allografts $(1.43 \pm 0.98)$ and autografts $(0.86 \pm 0.69)$; no statistical diferences existed between them. An increased level of metalloproteinases and TIMP-2 expression was obreved only after allografts with statistical differences among the allograft group, the autograft group nad the control group $(p<0.05)$.

Conclusions: Our findings suggest that the meniscal graft does not protect the hyaline cartilage against excessive apoptosis. The results of experimantal studies on humans indicate the need to device a method of apoptosis inhibition in the hyaline cartilage to improve long-term results of meniscal transplantation.

Key words: hyaline cartilage, apoptosis, metalloproteinases, viable meniscal transplantation.

\section{Introduction}

The purpose of the allogeneic meniscal graft is the protection of the hyaline cartilage against excessive wear and development of early degenerative changes [1-4]. Previous experimental studies suggest incomplete protection of the cartilage by the meniscal graft [3, 5-7]. Macro- and microscopic studies of the hyaline cartilage after meniscal grafting show degenerative changes [8-12]. The causes of this fact are not yet fully discovered. Due to the fact that the condition of the cartilage is one of the main factors determining the outcome of the meniscal graft, the explanation of the biological phenomena taking place in the hyaline cartilage and leading to its degeneration is of key importance $[1,13]$.
Corresponding author: Tomasz J. Zwierzchowski MD, PhD Arthroscopy and Sports Traumatology Clinic Orthopedic Department Medical University of Lodz 113 Zeromskiego St 90-549 Lodz, Poland Phone: +48509508781 Fax: +48426393539 E-mail: zwierzchowski.jacek @gmail.com 
The degenerative changes observed after meniscal grafting suggest that they may result from cartilage homeostasis disorders. Nowadays not much is known about the mechanisms regulating homeostasis of the hyaline cartilage after meniscal grafts $[1,3,13]$.

In normal hyaline cartilage programmed cell death (apoptosis) and metalloproteinases play a significant role in maintaining homeostasis [14-19]. Apoptosis - a highly regulated and controlled process of cell death - participates in homeostasis by eliminating wasted or mutated cells $[15,16,19,20]$. Metalloproteinases, zinc-dependent endopeptidases, on the other hand participate in cellular matrix transformations, providing the equilibrium between production and degradation of the extracellular matrix $[17,18]$. Among numerous metalloproteinases, a significant role in cellular matrix transformations is played by collagenase-1 (MMP-1), degrading type-II collagen and stromelysin-1 (MMP-3), degrading proteoglycans [21-23]. The matrix metalloproteinases are constrained by 4 tissue inhibitors (TIMPS). TIMP-2, apart from the inhibition of a wide spectrum of metalloproteinases, shows various biological effects important for the biology of hyaline cartilage [17, 18, 24].

Excessive apoptosis and the increase of matrix metalloproteinase expression are observed in various pathological conditions, including degenerative changes of the cartilage. These are not only determinants, but may also correspond to the development of degenerative changes [20, 25-30].

The use of an ideal transplant material such as fresh menisci and their application as an autogenous graft will enable the objectivization of the phenomena taking place in the joint cartilage after meniscal grafting [7, 31-33]. The incubation of fresh menisci in tissue culture conditions will enable experimental conditions to imitate clinical conditions $[34,35]$.

The purpose of this comparative study was to assess apoptosis and expression of MMP-1, MMP-3 and TIMP-2 in rabbit tibial cartilage 6 months after viable medial meniscal autografts and allografts and a control group.

\section{Material and methods}

\section{Study design}

Twenty-two white male New Zealand rabbits, body weight 3500-4000 g, were chosen for the study. All procedures were performed with the consent of the Local Ethical Committee for Experiments on Animals (Number $Ł / B D / 254$ ). Two study groups were distinguished: 8 animals with allografts and 8 with autografts. The third group was a control group -6 rabbits which only underwent arthrotomy (sham operation) without any other procedures on the meniscus. The excised left knee medial menisci (from 16 animals) were stored under tissue culture conditions. After 2 weeks' storage, from among the menisci prepared in this way, 8 rabbits were implanted with their own menisci into the left knee joint - autografts. The remaining 8 animals were implanted with donor menisci allografts. Two rabbits (1 with autograft and 1 with allograft) developed infective arthritis and were excluded from the study. All animals were euthanised after 6 months; the tibial cartilage was used for immunohistochemical examinations.

The apoptosis and immunohistochemistry evaluation was performed by a single observer, who was blinded to the experimental groups.

\section{Surgical procedure}

All surgical procedures were performed by the same surgeon. The rabbits were premedicated with intravenous medetomidyne at a dose of $0.01 \mathrm{ml} / \mathrm{kg}$ b.w. and atropine at $0.05 \mathrm{mg} / \mathrm{kg}$ b.w. To maintain anesthesia, Bioketan (ketamine) was administered as an intramuscular dose of $3 \mathrm{mg} / \mathrm{kg}$ b.w. Antisedan at a dose of $0.01 \mathrm{ml} / \mathrm{kg}$ b.w. was used to wake the animals. Under aseptic conditions, a skin incision of about $25 \mathrm{~mm}$ was performed on the medial side, and two medial anterior and posterior openings were made in the left joint capsule. The medial meniscus was excised according to the modified Shibuya technique without cutting the collateral tibial ligament [12]. The wound was closed in layers. The excised meniscus was placed in a tissue culture bottle (Nunc) with Dulbecco's modified Eagle medium (DMEM) : Ham F12 (1: 1) (Gibco) with the addition of $10 \%$ fetal calf serum (Sigma) and $1 \%$ penicillin, streptomycin and Fungizone (Gibco). The containers were stored in an incubator for 2 weeks, at $37^{\circ} \mathrm{C}$ in an atmosphere of $5 \% \mathrm{CO}_{2}$ and $95 \%$ air. Every 3 days, the bottles and medium were exchanged. From among the menisci prepared in this way, 8 rabbits were implanted with their own menisci into the left knee joint - autografts. The remaining 8 animals were implanted with donor menisci allografts. The medial meniscus was implanted according to a modified technique of Sommerlath and Gillquist [36]. After each procedure, antibiotic (enrofloxacin) was administered for prophylactic purposes at an intramuscular dose of $10 \mathrm{mg} / \mathrm{kg}$ b.w. every $12 \mathrm{~h}$ for 3 days. After surgery, the animals received butorphanol subcutaneously ( $0.3 \mathrm{mg} / \mathrm{kg}$ b.w.). No plaster immobilization was applied but the rabbits were allowed to move freely in standard cages. The animals' behavior was observed systematically. Postoperative wounds healed per primam, except two animals, which were excluded from the study.

After 6 months, the animals were euthanised by administering a lethal dose of pentabarbitone sodium intravenously. The left knee joint was opened. 
The hyaline cartilage from the medial tibial compartment was carefully excised. For the immunohistochemical studies, hyaline cartilage from meniscuscovered areas was harvested.

\section{Apoptosis detection}

The apoptosis was evaluated using the TUNEL method. Formalin-fixed, paraffin-embedded tissue sections were mounted onto SuperFrost slides and deparaffinized with xylene, then washed with alcohol and transferred to distilled water. Afterwards TUNEL Apoptosis Detection Kit (DNA Fragmentation/Fluorescence Staining; No. 17-141; Upstate NY) prepared according to the instructions of the manufacturer was used. In each specimen immunofluorescence was recorded semiquantitatively according to Durand et al. in 3-7 adjacent high power fields (magn. $400 \times$ ) and graded 0 (immunofluorescence not detectable), 1 (weak immunofluorescence), 2 (moderate immunofluorescence) and 3 (strong immunofluorescence) [37]. Then the mean grade was calculated.

In the selected samples, apoptosis was confirmed by evaluating its morphological exponents in hematoxylin and eosin (H\&E) stained specimens under a light microscope.

\section{Immunohistochemistry}

Paraffin sections were mounted onto SuperFrost slides, deparaffinized, then treated in a microwave oven in a solution of EDTA, pH 8.0 for $20 \mathrm{~min}$ $(2 \times 360 \mathrm{~W}-5 \mathrm{~min}, 2 \times 180 \mathrm{~W}-5 \mathrm{~min})$ and transferred to distilled water. Endogenous peroxidase activity was blocked by $0.3 \%$ hydrogen peroxide in distilled water for $30 \mathrm{~min}$, and then the sections were rinsed with Tris-buffered saline (TBS, DakoCytomation, Denmark) and incubated all night at $4^{\circ} \mathrm{C}$, with the following mouse monoclonal antibodies: anti-MMP-1 (Chemicon, MAB 3307, Germany, dilution 1 : 200), anti-MMP-3 (Chemicon, MAB 3312, Germany, dilution $1: 250$ ) and rabbit monoclonal antibody against TIMP-2 (Chemicon, AB 19029, Germany, dilution $1: 300$ ). Afterwards appropriate EnVision+Systems-HRP (DakoCytomation, Denmark) prepared according to the instructions of the manufacturer were used. Visualization was performed by incubating the sections in a solution of 3,3'diaminobenzidine (DakoCytomation, Denmark). After washing, the sections were counter-stained with hematoxylin and cover-slipped. For each antibody and for each sample a negative control was processed.

Negative controls were carried out by incubation in the absence of the primary antibody and always yielded negative results.

Staining intensities of MMP-1, MMP-3 and TIMP-2 were recorded semiquantitatively according to Choi et al. in 3-7 consecutive high power fields, graded
0 (negative), 1 (weakly positive), 2 (moderately positive) and 3 (strongly positive) [38]. Then the mean grade was calculated.

\section{Statistical analysis}

All values were expressed as the mean \pm SD (standard deviation). All the variables were treated as measurable ones. The Shapiro-Wilk normality test served as a basis for deciding on a parametric or non-parametric statistical test. In all groups there was no reason to reject the hypothesis that the variable has a normal distribution, except for apoptosis, which does not appear in the control group. The Shapiro-Wilk normality test results enabled the application of parametric methods in the subsequent calculations. Next Levene's test was conducted, which rejected the hypothesis of homogeneity of variances for apoptosis and immunohistochemistry. The Mann-Whitney test was used for evaluation of statistical differences between experimental and control groups. Value of $p<0.05$ was considered to be the level of statistical significance.

\section{Results}

\section{Apoptosis evaluation}

The TUNEL test revealed increased tibial cartilage chondrocyte apoptosis in both the autogenous and allogeneic graft group (Figures $1 \mathrm{~A}$ and $2 \mathrm{~A}$ ). The statistical analysis did not show any significant differences between groups. A statistical difference $(p<0.05)$ was observed between the control group, in which we did not observe apoptosis, and the allograft and autograft groups. The mean values of apoptosis for the study groups and control group are given in Table I.

\section{Immunohistochemical evaluation}

The immunoexpression of MMP-1, MMP-3 and TIMP-2 was significantly increased in the allografts (Figures 2 B-D), as compared to the autografts and controls $(p<0.05)$ (Figures $1 \mathrm{~B}-\mathrm{D})$ ). The difference of the MMP-1, MMP-3 and TIMP-2 immunoexpression between autografts and controls was weak and not significant. The mean values of the immunoexpression of MMP-1, MMP-3 and TIMP-2 are given in Table I.

\section{Discussion}

A review of the literature indicates that this is the first study to assess apoptosis and metalloproteinase expression in the articular cartilage after meniscus transplantation. Several conducted experimental studies evaluated the knee joint cartilage after implantation of meniscal grafts, assessing their potential chondroprotective capabilities [3]. Mikic et al. observed degenerative changes of the ti- 

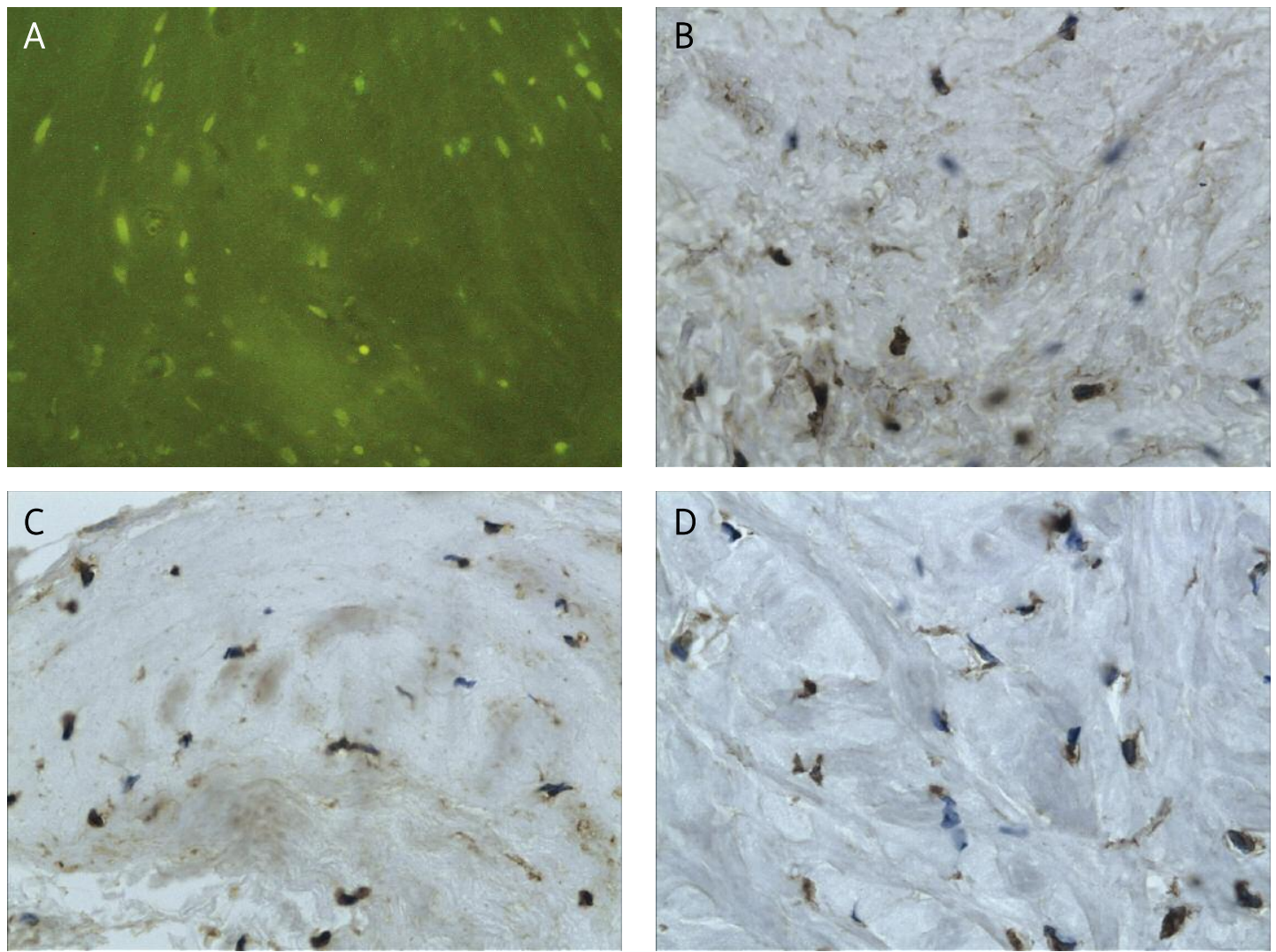

Figure 1. Hyaline cartilage of the tibia after viable meniscal autograft: A - TUNEL staining demonstrating apoptotic cells (JPEG), magn. $200 \times, \mathrm{B}-$ immunohistochemical staining of MMP-1 (JPEG), magn. $400 \times, \mathrm{C}$ - Immunohistochemical staining of MMP-3 (JPEG), magn. $400 \times$, D - immunohistochemical staining of TIMP-2 (JPEG), magn. $400 \times$

Table I. The immunoexpression of apoptosis, MMP-1, MMP-3 and TIMP-2 in the study groups and the control group

\begin{tabular}{|lcccc|}
\hline & Apoptosis & MMP-1 & MMP-3 & TIMP-2 \\
\hline Control & $0.00 \pm 0.00$ & $0.15 \pm 0.15$ & $0.22 \pm 0.06$ & $0.26 \pm 0.09$ \\
\hline Autografts & $0.86 \pm 0.69^{\ddagger}$ & $0.16 \pm 0.19$ & $0.26 \pm 0.30$ & $016 \pm 0.19$ \\
\hline Allografts & $1.43 \pm 0.98^{\S}$ & $2.25 \pm 0.59^{\S \dagger}$ & $2.29 \pm 0.63^{\S \dagger}$ & $1.40 \pm 0.58^{\S \dagger}$ \\
\hline
\end{tabular}

Mean value \pm standard deviation (SD), ${ }^{8}$ statistically significant differences between control and allografts $(p<0.05)$, ${ }^{1}$ statistically significant differences between auto- and allografts $(p<0.05)$, " statistically significant differences between autografts and control $(p<0.05)$

bial cartilage in dogs after fresh and frozen menisci grafting $[9,10]$. Degenerative changes in $25 \%$ of rabbits were noted by Shibuya after implanting cryopreserved meniscal allografts [12]. Rijk et al. observed that immediate allogeneic grafting of fresh meniscus in rabbits causes fewer degenerative changes than delayed grafting [11]. In the case of delayed transplantation in the rabbit, there is a chance of a partial withdrawal of cartilage degeneration, which was observed by Cummins et al. [8]. Studies by Mora et al. in sheep revealed the presence of degenerative changes after immediate fresh allogeneic grafting. According to various authors, the above observations prove that an allogeneic graft provides, albeit not full, cartilage protection [6]. The studies by Aagaard et al. in sheep confirmed that 6 months after immediate allogeneic grafting of fresh menisci, degenerative changes of the joint cartilage are seen. It indicates that the allogeneic meniscal graft reduces but does not completely prevent the degenerative changes [5]. Szomor et al. transplanted freshly harvested menisci in sheep and used them as allo- and autografts. The presence of degenerative changes of the joint cartilage 4 months after autogenous graft transplantation indicated that the meniscal graft does not provide complete protection of the joint cartilage [7]. The above-cited authors based their studies on macroscopic, histological and histochemical cartilage evaluation [5-12].

Our own studies of tibial hyaline cartilage are based on the evaluation of apoptosis and MMP-1, MMP-3 and TIMP-2 expression. Due to the fact that apoptosis and metalloproteinases are involved in hyaline cartilage homeostasis regulation and may 

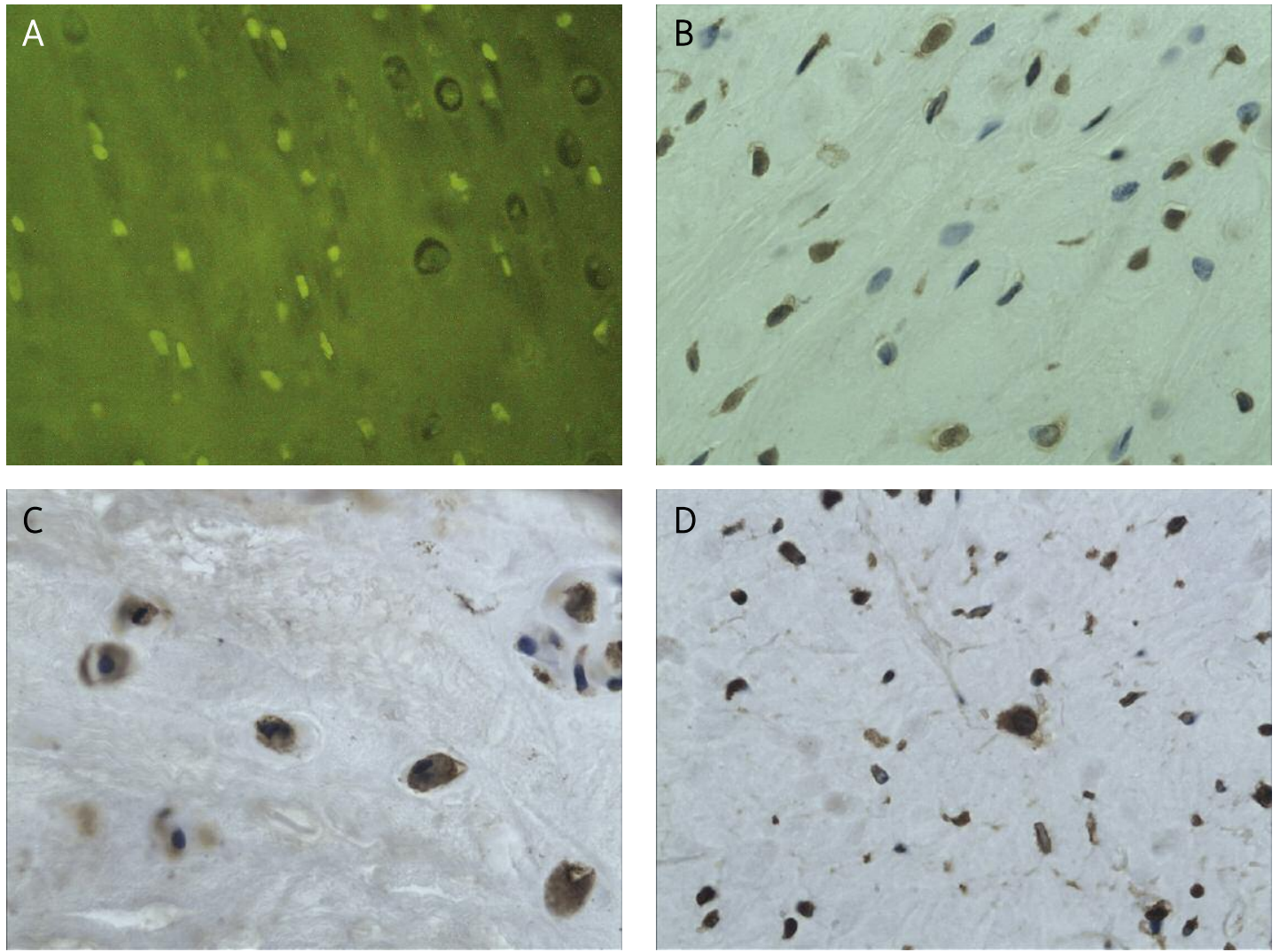

Figure 2. Hyaline cartilage of the tibia after viable meniscal allograft: A - TUNEL staining demonstrating apoptotic cells (JPEG), magn. $200 \times$, B - immunohistochemical staining of MMP-1 (JPEG), magn. $400 \times, C$-immunohistochemical staining of MMP-3 (JPEG), magn. 400 ×, D - immunohistochemical staining of TIMP-2 (JPEG), magn. 400×

correspond with cartilage destruction, our methodology will enable not only assessment of the joint cartilage, but also determination of the factors leading to the onset of degenerative changes [20-23, $25,26,29,30]$.

In our experimental studies we used only fresh menisci, which are thought to be the ultimate transplantation material both for experimental research and clinical practice $[1,5,6,8,9,11,31,34]$. In order to provide optimal protection of the joint cartilage, we transplanted fresh menisci as autogenous grafts in the same way as Szomor et al. and we compared them with fresh allografts [7]. In order to simulate clinical conditions we incubated the menisci in tissue culture for 2 weeks prior to transplantation [34]. Fresh menisci prepared in such a way have been successfully used in humans by Verdonk et al. [35]. The apoptosis evaluation and metalloproteinase expression tests were conducted on the part of the tibial cartilage found by Mikic et al. to suffer from the least intensive degenerative changes, that is in the area covered by the meniscal graft $[9,10]$. Those less advanced degenerative changes suggest their primary phase and enable one to search for the factors that cause them [30].

Our studies revealed excessive tibial hyaline cartilage chondrocyte apoptosis 6 months after both allogeneic and autogenous grafting of fresh, tis- sue culture conditioned medial menisci in the rabbit.

Hyaline cartilage is a tissue completely lacking blood vessels, which is the reason it cannot be penetrated by migrating cells, including those of the inflammatory response. Due to that fact, the chondrocytes are the only cells undergoing apoptosis. For our research we used the widely accepted TUNEL method, then we confirmed the results evaluating the morphological signs of apoptosis in cartilage H\&E stained specimens [20, 25].

Excessive apoptosis in the joint cartilage after meniscal transplantation should be considered a detrimental phenomenon. Apoptosis plays a role in the development of cartilage degeneration, and its intensity correlates with the severity of degenerative changes [20, 25, 28, 29]. Our study indicates that even a perfect transplant material, such as fresh autogenous menisci, does not prevent the joint cartilage from undergoing excessive chondrocyte apoptosis. The lack of apoptosis in the control group proves that sole knee joint arthrotomy does not influence the induction of hyaline cartilage chondrocyte apoptosis. Apoptosis is rarely detected in normal native cartilage $[15,29]$.

Hashimoto et al. presented a model mechanism linking disturbed apoptosis and excessive metalloproteinase expression [28]. 
The MMP-1, MMP-3 and TIMP-2 expression evaluation showed only a slight increase in the joint cartilage in the control and autogenous meniscal graft groups. The scant MMP-1, MMP-3 and TIMP-2 expression in the cartilage after autogenous grafting confirms their role in extracellular matrix transformations similar to the level found in natural cartilage - the control group.

Increased MMP-1, MMP-3 and TIMP-2 expression was present in the tibial hyaline cartilage after allogeneic grafting. Excessive metalloproteinase expression, according to Deier, indicates irreversible extracellular matrix changes [27]. It confirms the significant role of MMP-1 and MMP-3 in the development of hyaline cartilage degenerative changes after meniscal allograft transplantation. The increased expression of MMP-3 suggests the possibility of complex homeostasis disturbance in the cartilage after allogeneic meniscal grafting. Stromelysin 1, apart from the degradation of the proteoglycans, takes part in an alternative way of MMP-9 activation. This alternative way of MMP-9 activation takes place only in hyaline cartilage degenerative changes [27]. Also the excessive expression of TIMP-2 proves the complexity of cartilage disturbance. This tissue metalloproteinase inhibitor, apart from inhibiting a broad spectrum of metalloproteinases, also activates proMMP-2 [24].

There are limitations of our studies due to the fact that the results refer to experiments in rabbits. The apoptosis and metalloproteinase expression evaluation is limited to 6 months of observation. The control group consisted of animals after knee joint arthrotomies. Because apoptosis is claimed to be an early symptom of the degenerative changes and we found major degeneration with large areas of subchondral layer exposure in the knees after meniscectomy, we thought the apoptosis level evaluation in these knees to be of no use. The search for the causes of the fact that the transplanted menisci do not fully protect the joint cartilage made us relate our findings in the cartilage after meniscal grafting only to the knees which underwent arthrotomy. The sole MMP-1, MMP-3 and TIMP-2 evaluation with no TIMP-2 expression assessment and semiquantitative immunohistochemical tests methodology are limitations of our study.

The conducted experimental work proves the statement that any meniscal graft, even an autogenous graft, does not guard the joint cartilage against excessive apoptosis. The excessive apoptosis in the cartilage after both types of meniscal grafting may explain other authors' observations of incomplete cartilage protection after meniscal grafting [5-7]. Meniscal allografts do not protect the extracellular matrix against changes. This may lead to degenerative changes of the knee joint.

Apoptosis, in contrast to necrosis, is a highly regulated phenomenon, which makes it possible to be controlled [39]. Our observations of the cartilage after autogenous meniscal transplantation indicate the need of further research in excessive chondrocyte apoptosis inhibiting factors and methods.

If our present results in rabbits are to be related to the clinical outcomes of meniscal grafting in humans, the development and implementation of anti-apoptotic therapy in hyaline cartilage should be considered to improve late outcomes.

\section{Acknowledgments}

This study was supported by the research project of Medical University of Lodz: 502-17-263.

\section{References}

1. Lubowitz JH, Verdonk PC, Reid JB 3rd, Verdonk R. Meniscus allograft transplantation: a current concepts review. Knee Surg Sports Traumatol Arthrosc 2007; 15: 476-92.

2. Rijk PC. Meniscal allograft transplantation - part I: background, results, graft selection and preservation, and surgical considerations. Arthroscopy 2004; 20: 728-43.

3. Rijk PC. Meniscal allograft transplantation - part II: alternative treatments, effects on articular cartilage, and future directions. Arthroscopy 2004; 20: 851-9.

4. Verdonk R. Meniscal transplantation. Acta Orthop Belg 2002; 68: 118-27.

5. Aagaard H, Jørgensen U, Bojsen-Møller F. Immediate versus delayed meniscal allograft transplantation in sheep. Clin Orthop Relat Res 2003; 406: 218-27.

6. Mora G, Alvarez E, Ripalda P, Forriol F. Articular cartilage degeneration after frozen meniscus and Achilles tendon allograft transplantation: experimental study in sheep. Arthroscopy 2003; 19: 833-41.

7. Szomor ZL, Martin TE, Bonar F, Murrell GA. The protective effects of meniscal transplantation on cartilage. An experimental study in sheep. J Bone Joint Surg Am 2000; 82: 80-8.

8. Cummins JF, Mansour JN, Howe Z, Allan DG. Meniscal transplantation and degenerative articular change: an experimental study in the rabbit. Arthroscopy 1997; 13: 485-91.

9. Mikic ZD, Brankov MZ, Tubic MV, Lazetic AB. Allograft meniscus transplantation in the dog. Acta Orthop Scand 1993; 64: 329-32.

10. Mikic ZD, Brankov MZ, Tubic MV, Lazetic AB. Transplantation of fresh-frozen menisci: an experimental study in dogs. Arthroscopy 1997; 13: 579-83.

11. Rijk PC, Tigchelaar-Gutter W, Bernoski FP, Van Noorden CJ. Histologic changes in articular cartilage after medial meniscus replacement in rabbits. Arthroscopy 2004; 20: 911-17.

12. Shibuya S. Meniscus transplantation using a cryopreserved allograft. Histological and ultrastructural study of the transplanted meniscus. J Orthop Sci 1999; 4: 135-41.

13. Messner K, Kohn D, Verdonk R. Future research in meniscal replacement. Scand J Med Sci Sports 1999; 9: 181-3.

14. Fu J, Wang P, Zhang X, et al. Myeloma cells inhibit osteogenic differentiation of mesenchymal stem cells and kill osteoblasts via TRAlL-induced apoptosis. Arch Med Sci 2010; 6: 496-504.

15. Kim HT, Teng MS, Dang AC. Chondrocyte apoptosis: implications for osteochondral allograft transplantation. Clin Orthop Relat Res 2008; 466: 1819-25.

16. Majno G, Joris I. Apoptosis, oncosis, and necrosis. An overview of cell death. Am J Pathol 1995; 146: 3-15. 
17. Nagase H, Visse R, Murphy G. Structure and function of matrix metalloproteinases and TIMPs. Cardiovasc Res 2006; 69: 562-73.

18. Visse R, Nagase H. Matrix metalloproteinases and tissue inhibitors of metalloproteinases: structure, function, and biochemistry. Circ Res 2003; 92: 827-39.

19. Wyllie AH, Kerr JF, Currie AR. Cell death: the significance of apoptosis. Int Rev Cytol 1980; 68: 251-306.

20. Aigner T, Kim HA. Apoptosis and cellular vitality: issues in osteoarthritic cartilage degeneration. Arthritis Rheum 2002; 46: 1986-96.

21. Takahashi K, Goomer RS, Harwood F, Kubo T, Hirasawa Y, Amiel D. The effects of hyaluronan on matrix metalloproteinase-3 (MMP-3), interleukin-1beta(IL-1beta), and tissue inhibitor of metalloproteinase-1 (TIMP-1) gene expression during the development of osteoarthritis. Osteoarthritis Cart 1999; 7: 182-90.

22. Tsuzaki M, Guyton G, Garrett W, et al. IL-1 beta induces COX2, MMP-1, -3 and -13, ADAMTS-4, IL-1 beta and IL- 6 in human tendon cells. J Orthop Res 2003; 21: 256-64.

23. Wu W, Billinghurst RC, Pidoux I, et al. Sites of collagenase cleavage and denaturation of type II collagen in aging and osteoarthritic articular cartilage and their relationship to the distribution of matrix metalloproteinase 1 and matrix metalloproteinase 13. Arthritis Rheum 2002; 46: 2087-94.

24. Baker AH, Edwards DR, Murphy G. Metalloproteinase inhibitors: biological actions and therapeutic opportunities. J Cell Sci 2002; 115: 3719-27.

25. Blanco FJ, Guitian R, Vázquez-Martul E, de Toro FJ, Galdo F. Osteoarthritis chondrocytes die by apoptosis. A possible pathway for osteoarthritis pathology. Arthritis Rheum 1998; 41: 284-9.

26. Bluteau G, Conrozier T, Mathieu P, Vignon E, Herbage D, Mallein-Gerin F. Matrix metalloproteinase-1, -3, -13 and aggrecanase- 1 and -2 are differentially expressed in experimental osteoarthritis. Biochim Biophys Acta 200; 1526: 147-58.

27. Dreier R, Grässel S, Fuchs S, Schaumburger J, Bruckner P. Pro-MMP-9 is a specific macrophage product and is activated by osteoarthritic chondrocytes via MMP-3 or a MT1 MMP/MMP-13 cascade. Exp Cell Res 2004; 297: 303-12.

28. Hashimoto S, Takahashi K, Amiel D, Coutts RD, Lotz M. Chondrocyte apoptosis and nitric oxide production during experimentally induced osteoarthritis. Arthritis Rheum 1998; 41: 1266-74.

29. Hashimoto S, Ochs RL, Komiya S, Lotz M. Linkage of chondrocyte apoptosis and cartilage degradation in human osteoarthritis. Arthritis Rheum 1998; 41: 1632-8.

30. Pennock AT, Robertson CM, Emmerson BC, Harwood FL, Amiel D. Role of apoptotic and matrix-degrading genes in articular cartilage and meniscus of mature and aged rabbits during development of osteoarthritis. Arthitis Rheum 2007; 56: 1529-36.

31. Felix NA, Paulos LE. Current status of meniscal transplantation. Knee 2003; 10: 13-7.

32. Jackson DW, McDevitt CA, Simon TM, Arnoczky SP, Atwell EA Silvino NJ. Meniscal transplantation using fresh and cryopreserved allografts. An experimental study in goats. Am J Sports Med 1992; 20: 644-56.

33. Stone KR, Rodkey WG, McKinney LA, Steadman JR. Autogenous replacement of the meniscus cartilage: analysis of results and mechanisms of failure. Arthroscopy 1995; 11: 395-400.

34. Verdonk R. Alternative treatments for meniscal injuries. J Bone Joint Surg Br 1997; 79: 866-73.

35. Verdonk PC, Demurie A, Almqvist KF, Veys EM, Verbruggen G, Verdonk R. Transplantation of viable meniscal allograft. Survivorship analysis and clinical outcome of one hundred cases. J Bone Joint Surg Am 2005; 87: 715-24.
36. Sommerlath K, Gillquist J. The effects of an artificial meniscus substitute in a knee joint with a resected anterior cruciate ligament. An experimental study in rabbits. Clin Orthop 1993; 289: 276-84.

37. Durand E, Scoazec A, Lafont A, et al. In vivo induction of endothelial apoptosis leads to vessel thrombosis and endothelial denudation: a clue to the understanding of the mechanisms of thrombotic plaque erosion. Circulation 2004; 109: 2503-6.

38. Choi HR, Kondo S, Hirose K, Ishiguro N, Hasegawa Y, Iwata $\mathrm{H}$. Expression and enzymatic activity of MMP-2 during healing process of the acute supraspinatus tendon tear in rabbits. J Orthop Res 2002; 20: 927-33.

39. D'Lima D, Hermida J, Hashimoto S, Colwell C, Lotz M. Caspase inhibitors reduce severity of cartilage lesions in experimental osteoarthritis. Arthritis Rheum 2006; 54: 1814-21. 